\title{
Alveolar Macrophages Can Control Respiratory Syncytial Virus Infection in the Absence of Type I Interferons
}

\author{
Spyridon Makris ${ }^{\mathrm{a}}$ Monika Bajorek ${ }^{\mathrm{b}}$ Fiona J. Culley ${ }^{\mathrm{a}}$ Michelle Goritzka $^{\mathrm{a}}$ \\ Cecilia Johansson ${ }^{\mathrm{a}}$ \\ ${ }^{a}$ Respiratory Infections Section, St. Mary's Campus, National Heart and Lung Institute, and bepartment of \\ Medicine, Imperial College London, London, UK
}

\section{Key Words}

Macrophages · Type I interferons · Pattern recognition receptors

\begin{abstract}
Respiratory syncytial virus (RSV) is a common cause of lower respiratory tract infections. Immunity to RSV is initiated upon detection of the virus by pattern recognition receptors, such as RIG-I-like receptors. RIG-I-like receptors signal via MAVS to induce the synthesis of proinflammatory mediators, including type l interferons (IFNs), which trigger and shape antiviral responses and protect cells from infection. Alveolar macrophages (AMs) are amongst the first cells to encounter invading viruses and the ones producing type I IFNs. However, it is unclear whether IFNs act to prevent AMs from serving as vehicles for viral replication. In this study, primary AMs from MAVS (Mavs ${ }^{--}$)- or type I IFN receptor (Ifnar ${ }^{-1-}$ )-deficient mice were exposed to RSV ex vivo. Wild-type (wt) AMs but not Mavs $^{-/-}$and Ifnar ${ }^{-/-}$AMs produced inflammatory mediators in response to RSV. Furthermore, Mavs $^{-/-}$and Ifnar ${ }^{-/-}$ AMs accumulated more RSV proteins than wt AMs, but the infection was abortive. Thus, RIG-I-like receptor-MAVS and IFNAR signalling are important for the induction of proinflammatory mediators from AMs upon RSV infection, but this
\end{abstract}

\section{KARGER}

E-Mail karger@karger.com www.karger.com/jin

\section{() 2016 The Author(s) \\ Published by S. Karger AG, Basel 1662-811X/16/0085-0452\$0/0}

This article is licensed under the Creative Commons Attribution 4.0 International License (CC BY) (http://www.karger.com/Services/ OpenAccessLicense). Usage, derivative works and distribution are permitted provided that proper credit is given to the author and the original publisher. signalling is not central for controlling viral replication. The ability to restrict viral replication makes AMs ideal sensors of RSV infection and important initiators of immune responses in the lung.

(C) 2016 The Author(s)

Published by S. Karger AG, Basel

\section{Introduction}

Lung infections must be carefully managed by the host in order to prevent detrimental effects to gas exchange and lung tissue integrity. A pivotal balance must be maintained where the pathogen is eradicated rapidly while inflammation remains tightly controlled. Alveolar macrophages (AMs) reside in the alveoli at the airway epithelium and air interface, and account for over $95 \%$ of the leukocytes in the airways. These macrophages are a unique subset of mononuclear phagocytes in both phenotype and function $[1,2]$. They originate from fetal monocytes [3] and are believed to play a central role in maintaining tissue homeostasis and removing cellular debris $[1,2]$. AMs are also crucial for the initiation of immune responses against invading respiratory viruses [4-9].

Respiratory syncytial virus (RSV) can cause severe lower respiratory tract infections especially in infants, the 


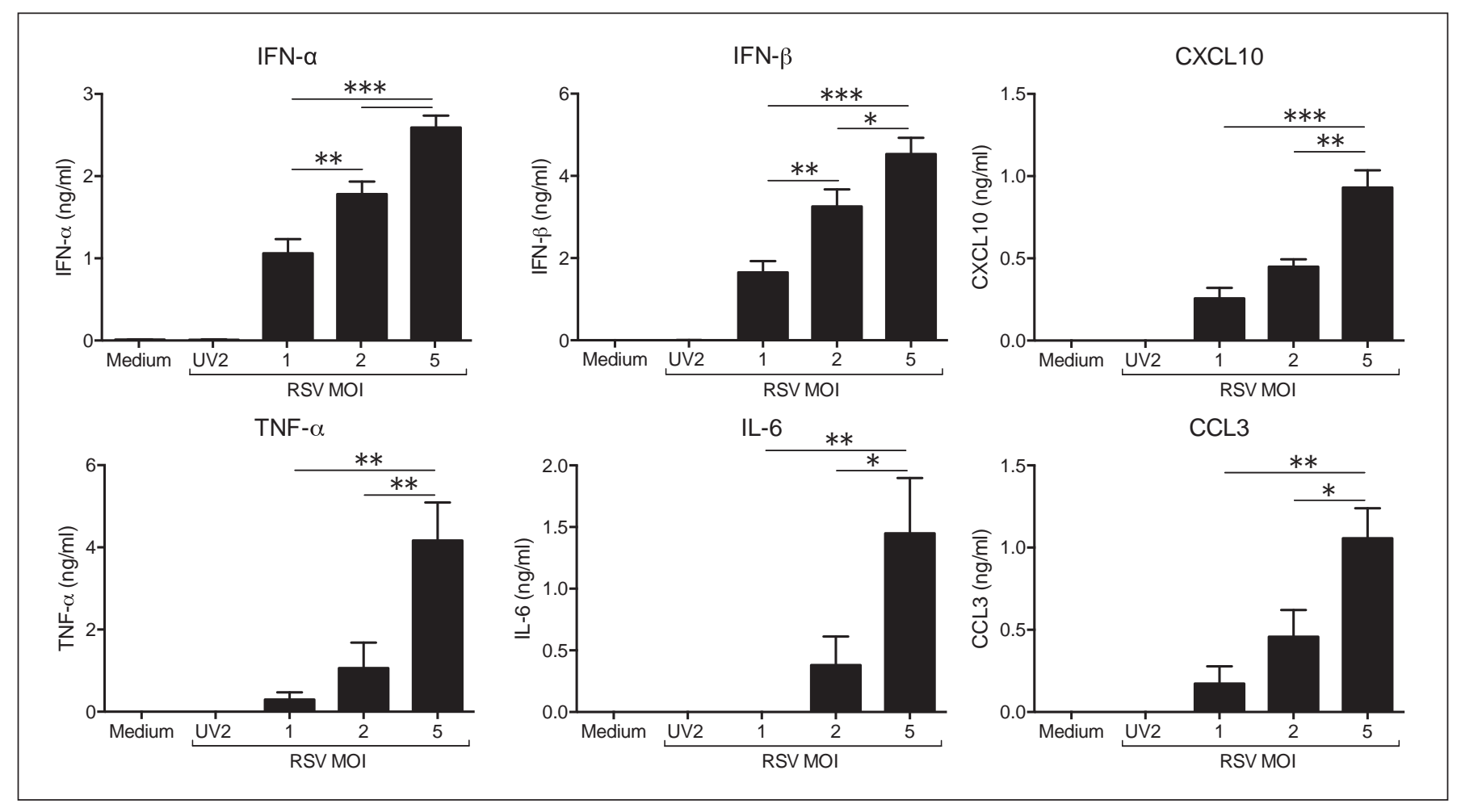

Fig. 1. AMs produce cytokines and chemokines after RSV exposure. Primary wt AMs were exposed for $20 \mathrm{~h}$ to medium or the indicated MOI of RSV or UV-RSV (MOI of 2; UV2). Secretion of IFN- $\alpha$, IFN- $\beta$, CXCL10, TNF- $\alpha$, IL-6, and CCL3 were detected in culture supernatant by Luminex. The data are shown as means \pm
SEM of 4-9 individual cultures pooled from 2-3 experiments. Statistical significance of differences between indicated groups was determined by one-way ANOVA. ${ }^{*} \mathrm{p}<0.05$; ${ }^{* *} \mathrm{p}<0.01$; $^{* * *} \mathrm{p}<$ 0.001 . immunocompromised, and the elderly [10]. Approximately $2-3 \%$ of RSV-infected children develop severe bronchiolitis/viral pneumonia and require hospitalisation [10], and many of these children are prone to recurrent wheeze and asthma-like symptoms in their adolescence [11]. Several studies suggest that the innate immune response is indispensable for the lung to maintain its function while clearing RSV [10]. RSV is recognized by pattern recognition receptors, which induce the production of type I interferons (IFNs) and other cytokines that enhance the antiviral responses [12].

Type I IFNs exert both cell-intrinsic effects, which limit viral replication, and cell-extrinsic antiviral effects, such as recruitment and activation of immune cells [12, 13]. Work from our group showed that during RSV infection, type I IFNs have a central role in both interfering with viral replication and driving lung inflammation during RSV infection $[4,14]$. AMs are the main producers of type I IFNs in response to RSV and this production is initiated via recognition of RSV by cytosolic MAVS-coupled pattern recognition receptors $[4,15]$.

Although studies suggest that AMs are early producers of inflammatory mediators, there is very little evidence as to how this production is initiated and especially how type I IFNs contribute to viral control in AMs. We therefore studied primary murine AMs deficient in MAVS and IFNAR1 exposed to RSV ex vivo. Our results show that AMs can produce several cytokines and chemokines after exposure to RSV and that this production is dependent on type I IFNs. However, AMs are not a source of all mediators known to be important early after RSV infection. Furthermore, we found that although the viral replication was abortive, transcription of RSV genes in both MAVSand IFNAR1-deficient AMs was increased in comparison to wild-type (wt) AMs. Thus, AMs efficiently control RSV infection and are an important source of cytokines and chemokines during RSV infection, highlighting their role as important guards of the lower airways. 


\section{Materials and Methods}

\section{Mice}

C57BL/6 mice were purchased from Charles River or Harlan, $\mathrm{UK}$, and Ifnar ${ }^{-/-}$mice on a C56BL/6 background were obtained from C. Reis e Sousa, The Francis Crick Institute, UK. Ifna $6^{8 f p}$ $\mathrm{Mavs}^{-1-}$ mice (obtained from S. Akira, Japan) were screened to ensure the genotype was maintained and are designated as $\mathrm{Mavs}^{-/-}$to denote the fact that the mice may or may not have a copy of the gfp insert at the Ifna6 locus. All mice were bred and maintained in pathogen-free conditions, and gender and age-matched mice aged 8-14 weeks were used for each experiment. All animal experiments were reviewed and approved by the Animal Welfare and Ethical Review Board (AWERB) within Imperial College London and approved by the UK Home Office in accordance with the Animals (Scientific Procedures) Act 1986 and the ARRIVE guidelines.

\section{Isolation of AMs}

Mice were sacrificed and primary AMs were collected by bronchoalveolar lavage through flushing the lungs 3 times with $1 \mathrm{ml}$ of PBS supplemented with 5 mM EDTA (Life Technologies). The lavage was repeated twice and AMs from several mice were pooled. The purity of the AMs was $>98 \%$ as determined by flow cytometry [4] and on cytospin counts. Collected cells were incubated in a flatbottom 96-well plate $\left(1.25 \times 10^{5}\right.$ cells/well) in complete DMEM (Invitrogen) containing 10\% FCS (heat-inactivated from Gibco), $2 \mathrm{mM}$ L-glutamine (Invitrogen), and 1,000 U/ml penicillin-streptomycin (Sigma-Aldrich) for $3 \mathrm{~h}$. After washing, the adherent cells were exposed to various stimuli (see below).

\section{Virus Infections, Cell Lines, and Innate Stimulation}

Plaque-purified human RSV (originally the A2 strain from ATCC, US) was grown in HEp2 cells [16]. Inactivation was performed by exposing the virus to UV light for $2 \mathrm{~min}$ (UV-RSV) in a CX-2000 UV cross-linker (UVP). The mouse epithelial cell line LA4 was grown in Ham's F12K medium containing 10\% FCS (heat-inactivated from Gibco), $2 \mathrm{mM}$ L-glutamine (Invitrogen), and 1,000 U/ml penicillin-streptomycin (Sigma-Aldrich).

AMs or LA4 cells were stimulated with different multiplicity of infections (MOIs) of RSV and UV-RSV, or to TLR ligands (R848 or LPS from InvivoGen; $1 \mathrm{ng} / \mathrm{ml}$ ), and incubated for $20 \mathrm{~h}$. The supernatant was collected and the cells were resuspended in Trizol for subsequent RNA extraction.

\section{RNA Isolation and $q P C R$}

RNA extraction from AMs or LA4 was performed using Trizol reagent (Invitrogen) according to manufacturer's instructions. The RNA then underwent DNase treatment according to manufacturer's instructions (Life Technologies). $0.5-1 \mu \mathrm{g}$ of RNA was reversetranscribed using the High Capacity RNA-to-cDNA kit according to the manufacturer's instructions (Applied Biosystems). qPCR was performed to quantify mRNA levels in AMs or LA4 cells. qPCR reactions for Ifnb, Ifng, Ifnl, Tnfa, Oas1a, Rsad2, Eif2ak2, and RSV L and $\mathrm{N}$ genes were performed using primers and probes previously described [4]. For the RSV, strand-specific qPCR was performed as previously described [17]. Briefly, RNA extracted from AMs or LA4 cells was converted using High Capacity cDNA reverse transcription Kit (Qiagen) with gene-specific primers for the positive- and negative-sense RSV nucleocaspid region RNA. Since the primers contain a tag sequence, a tag-specific primer was used in the qPCR
Table 1. Inflammatory mediators not or partially detected using Luminex, ELISA, or RT-PCR after $20 \mathrm{~h}$ of exposure of AMs to RSV MOI of 2

\begin{tabular}{|c|c|c|c|}
\hline \multirow{2}{*}{ Mediator } & \multicolumn{3}{|l|}{ Assay } \\
\hline & Luminex & ELISA & Gene expression \\
\hline IL-1 $\alpha$ & - & & + \\
\hline IL-1 $\beta$ & - & - & + \\
\hline IL-2 & - & & - \\
\hline IL-4 & - & & - \\
\hline IL-5 & - & & - \\
\hline IL-10 & - & & - \\
\hline IL12p40/p70 & - & & - \\
\hline IL-13 & - & & - \\
\hline IL-17 & - & & - \\
\hline IL-18 & & & - \\
\hline IFN- $\lambda$ & & & - \\
\hline $\mathrm{IFN}-\gamma$ & - & & - \\
\hline CXCL1 & - & - & + \\
\hline CCL2 & - & - & + \\
\hline CXCL9 & - & & + \\
\hline GM-CSF & - & & - \\
\hline VEGF & - & & - \\
\hline
\end{tabular}

Data are representative of at least two experiments.

[17]. Analysis was performed using the QuantiTect Probe PCR Master Mix (Qiagen) and the 7500 Fast Real-Time PCR System (Applied Biosystems). Results were normalised to levels of Gapdh, a housekeeping gene (Applied Biosystems). For absolute quantification, the exact number of copies of the gene of interest was calculated using a plasmid DNA standard curve for each gene. For relative quantification, the expression of RSV positive- and negative-sense strands [17], Ifna6, Cxcl10, Il6, Ccl3, Illa, Il1b, Il2, Il4, Il5, Il10, Il12p40/p70, Il13, Il17, Cxcl1, Ccl2, Cxcl9, Csf2, and Vegfa (all from Applied Biosystems) were expressed relatively to the expression of Gapdh. First, the $\Delta \mathrm{Ct}(\mathrm{Ct}=$ cycle threshold $)$ between the target gene and Gapdh for each sample was calculated. Then the expression was calculated as $2^{-\Delta \mathrm{Ct}}$. Analysis was performed using 7500 Fast System SDS Software (Applied Biosystems).

\section{Chemokine and Cytokine Detection}

Chemokines and cytokines were quantified by a Cytokines Mouse Magnetic 20-Plex Panel for Luminex (Life Technologies), and a ProcartaPlex mouse IFN- $\alpha /$ IFN- $\beta$ panel (eBioscience) according to the manufacturer's instructions and data were acquired using a Bio-Plex 200 system (Bio-Rad Laboratories, UK). The concentration of cytokines in each sample was determined according to the standard curve using the Bio-Plex 6 software (Bio-Rad Laboratories). The concentration of CXCL10, CCL2, and TNF- $\alpha$ was additionally measured using mouse DuoSet ELISA (R\&D) according to the manufacturer's instructions. IL- 6 was detected by ELISA using MP5-20F3 capture antibody and biotinylated MP5-32C11 detection antibody (both from BD Pharmingen). Data were acquired on a SpectraMax Plus plate reader (Molecular Devices) and analysed using SoftMax software (version 5.2). 


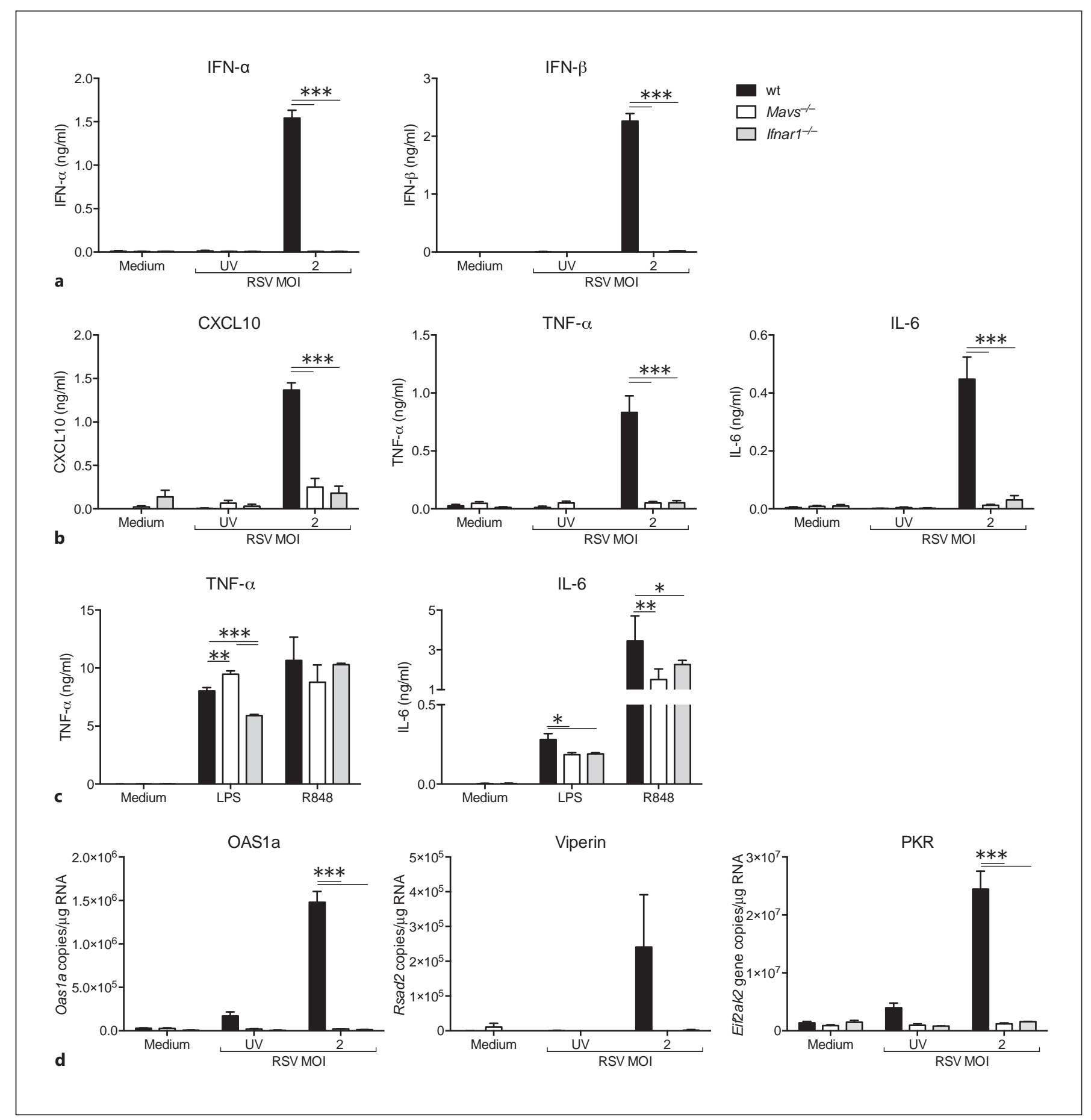

Fig. 2. Cytokines and chemokine production by AMs is dependent on MAVS and IFNAR1 signalling. Primary AMs from wt, $\mathrm{Mavs}^{-/-}$, and Ifnarl ${ }^{-1-}$ mice were exposed to medium or MOI of 2 of UV-RSV (UV) or RSV for $20 \mathrm{~h}$. IFN- $\alpha$ and IFN- $\beta$ (a) were detected in culture supernatant by Luminex and CXCL10, TNF- $\alpha$, and IL-6 (b) were detected in culture supernatant by ELISA. c Secretion of TNF- $\alpha$ and IL- 6 from primary AMs of the indicated genotypes after ex vivo exposure for $20 \mathrm{~h}$ to medium or $1 \mathrm{ng} / \mathrm{ml}$ of LPS or R848. d OAS1a, viperin, and PKR transcripts were detected

Type I IFN-Deficient AMs Can Control RSV Replication by RT-PCR after exposure to medium or MOI of 2 of UV-RSV (UV) or RSV for $20 \mathrm{~h}$. The data are shown as means \pm SEM of 4-6 cultures per stimulation pooled from 3 independent experiments (a), 4-15 cultures per stimulation pooled from 2-6 independent experiments (b), 4-8 cultures per stimulation pooled from 2-4 independent experiments (c), and as 3-4 individual RNA samples pooled from 3-4 independent experiments (d). Statistical significance of differences between indicated groups was determined by unpaired Student's t test. ${ }^{*} \mathrm{p}<0.05 ;{ }^{* *} \mathrm{p}<0.01$; $^{* *} \mathrm{p}<0.001$. 
Staining for Fluorescence Microscopy

$3.75 \times 10^{5}$ primary AMs were seeded in a 24 -well flat-bottom plate containing glass coverslips. The cells were allowed to adhere for $3 \mathrm{~h}$ before exposure to medium, RSV, or UV-RSV. After $21 \mathrm{~h}$ the cells were washed with PBS and fixed with $4 \%$ paraformaldehyde for 10 min at RT followed by blocking with 3\% BSA in $0.2 \%$ Triton X/PBS for a further $10 \mathrm{~min}$. The cells were stained with polyclonal anti-RSV (1:200; Abcam) or monoclonal anti-RSV N (1:300; Abcam) and anti-LAMP-1 (1:300; Abcam) antibodies for $1.5 \mathrm{~h}$ at RT. This was followed by staining with species-specific secondary antibodies conjugated to Alexa Fluor 488 or Alexa Fluor 568 (1:1,000; Invitrogen) for $45 \mathrm{~min}$ in the dark at RT. The coverslips were mounted onto glass slides with ProLong ${ }^{\circledR}$ Gold Antifade Mountant with DAPI (ThermoFisher Scientific). The Zeiss confocal laser-scanning microscope (LSM)-510 on a $100 \times / 1.4$ Plan-Apochromat oil lens was used for obtaining images of the cells. Analysis of the images was performed on Fiji, an open-source imaging processing software (ImageJ).

\section{Western Blot Analysis}

Lysates from $8 \times 10^{5}$ primary AMs extracted using RIPA Buffer (Sigma) containing cOmplete Protease Inhibitors (Mini; Roche). Briefly, the cells were resuspended in RIPA buffer and placed on a rotator at $4^{\circ} \mathrm{C}$ for $30 \mathrm{~min}$ before being centrifuged at $12,000 \mathrm{~g}$ for $10 \mathrm{~min}$ at $4^{\circ} \mathrm{C}$. The supernatant was quantified using the Peirce ${ }^{\mathrm{TM}}$ BCA protein assay kit (Thermo Scientific) following the manufacturer's instruction. For Western blots, $5 \mu \mathrm{g}$ of protein was added in each lane in a 1:1 dilution with Laemmli buffer containing bromophenol blue (Sigma). Proteins were loaded onto a 10-lane 4-12\% Bis-Tris gel (Novex) containing the MagicMark ${ }^{\mathrm{TM}} \mathrm{XP}$ Western Protein Standard (Invitrogen) markers. Gels were run in running buffer (NugePage MES SDS) before being transferred to a polyvinylidene difluoride filter paper sandwich with $0.45-\mu \mathrm{m}$ pore membrane (Novex) using Tris-glycine transferase (Novex). The membranes were blocked for $1 \mathrm{~h}$ and then exposed to a goat-antiRSV antibody (1:2,000; Abcam) or Direct-Blot ${ }^{\mathrm{TM}} \mathrm{HRP}$ anti- $\beta$-actin (1:1,000; BioLegend) at $4{ }^{\circ} \mathrm{C}$ overnight in blocking buffer (TBS containing $5 \%$ BSA and $0.01 \%$ TWEEN20). The membranes were then incubated with a secondary anti-goat HRP antibody $(1: 5,000$; Jackson ImmunoResearch) for $1 \mathrm{~h}$ before being developed using EZ-ECL (Biological Industries). The membrane was read using a Fusion FX reader (Vilber Lourmat) and analysis performed using the Vilber Lourmat software program.

\section{Statistical Analysis}

Statistical analysis was performed using GraphPad Prism 6 (GraphPad Software Inc.). Comparisons were performed using either an unpaired Student's t test or one-way ANOVA with Tukey's post hoc test. For all tests, $\mathrm{p}<0.05$ was considered significant.

Fig. 3. Accumulation of RSV proteins in $\mathrm{Mavs}^{-/-}$and Ifnarl $1^{-/-}$pri- $^{-}$ mary AMs. Primary AMs from wt, $\mathrm{Mavs}^{-/-}$, and Ifnarl ${ }^{-/-}$mice were exposed to medium or MOI of 2 of RSV or UV-RSV for $21 \mathrm{~h}$ before being fixed, stained using polyclonal-anti-RSV antibodies and DAPI, and analysed by fluorescence microscopy. a Representative brightfield images of medium-exposed AMs. Representative

\section{Results}

\section{AMs Produce an Array of Cytokines and Chemokines} after RSV Exposure

AMs have the ability to rapidly respond to invading pathogens including RSV [4]. To investigate which inflammatory mediators are produced by AMs after encountering RSV, primary AMs were exposed to RSV ex vivo and quantification of secreted mediators and mRNA was performed by Luminex and RT-PCR, respectively. Initially a time course (6-96 h) was performed and 18$20 \mathrm{~h}$ was determined as the optimum time point to study both cytokine mRNA and protein induction (data not shown). At $20 \mathrm{~h}$ after RSV exposure, primary AMs produced IFN- $\alpha$, IFN- $\beta$, CXCL10, TNF- $\alpha$, IL- 6 , and CCL 3 as detected both by Luminex (fig. 1) and mRNA (see online suppl. fig. S1; for all online suppl. material, see www. karger.com/doi/10.1159/000446824). This cytokine and chemokine response was not triggered by UV-RSV and was positively correlated with RSV dose (MOI; fig. 1; online suppl. fig. S1). An MOI of 2 was then chosen for subsequent experiments. Interestingly, there were several inflammatory mediators that primary AMs exposed ex vivo to RSV did not produce, such as IL- $1 \alpha$, IL-1 $\beta$, IL-2, IL-4, IL-5, IL-10, IL-12p40/p70, IL-13, IL-17, IL-18, IFN- $\lambda$, IFN- $\gamma$, CXCL1, CCL2, CXCL9, GM-CSF, and VEGF, as assessed by Luminex and ELISA (table 1). A weak mRNA signal but no protein was detected for Illa, Il1b, Cxcl1, Ccl2, and Cxcl9 (table 1). Thus, AMs can produce many but not all of the early mediators detected in vivo in response to RSV infection [14], suggesting that they, together with other cell types, contribute to inflammatory lung responses during viral infection.

\section{The Cytokine and Chemokine Production from AMs Depends on MAVS and IFNAR Signalling}

We used primary AMs from wt, Ifnar1 ${ }^{-/}$, and $\mathrm{Mavs}^{-1-}$ mice, and exposed them ex vivo to RSV MOI of 2. We found that both IFNAR- and MAVS-deficient AMs lacked the production of IFN- $\alpha$, IFN- $\beta$, CXCL10, IL-6, and TNF- $\alpha$ by AMs after RSV exposure (fig. 2a, b). As a images of UV-RSV-exposed primary AMs (b) or RSV-exposed primary AMs (c) where location of 'inclusion-like bodies' are presented by white arrows. Scale bar represents $10 \mu \mathrm{m}$. Images characterise the appearance of the majority of AMs from at least 4 independent experiments.

(For figure see next page.) 

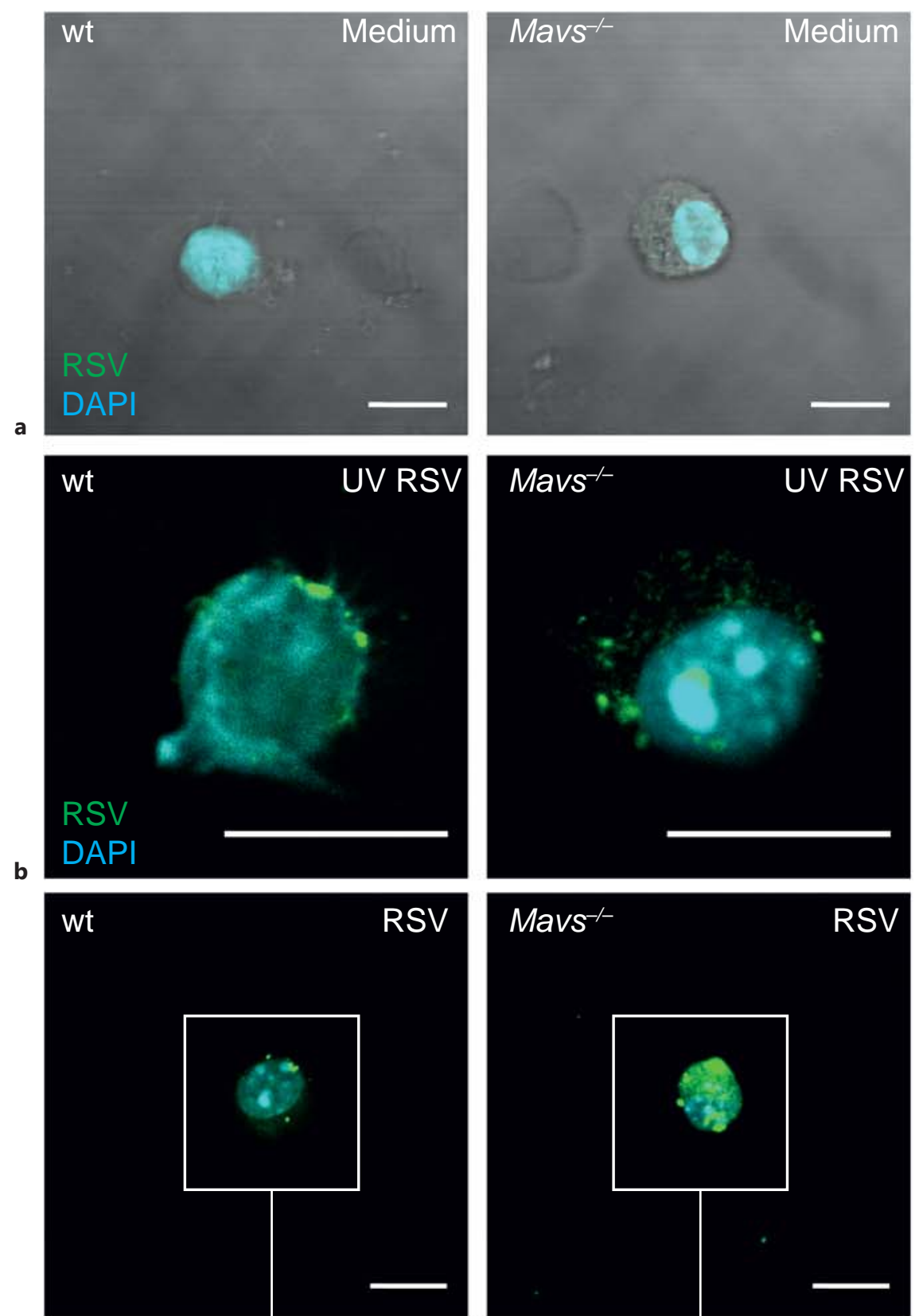

wt

RSV

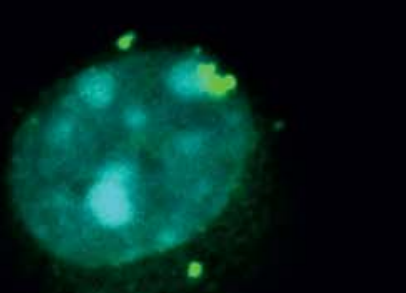

RSV

c
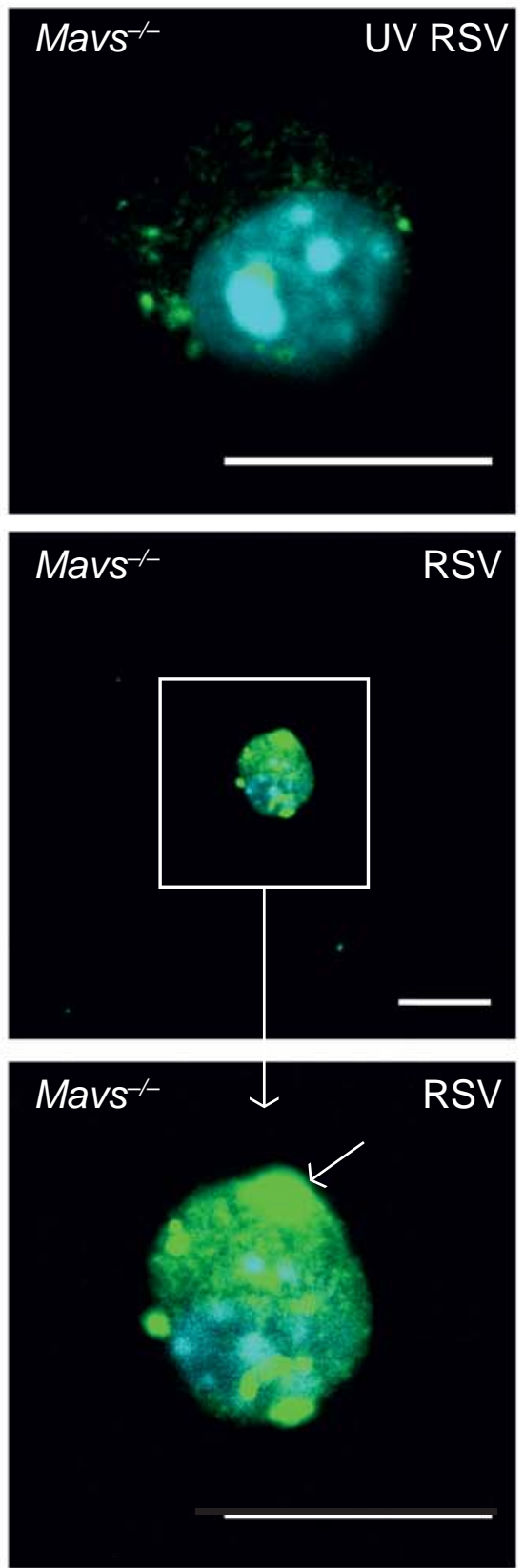

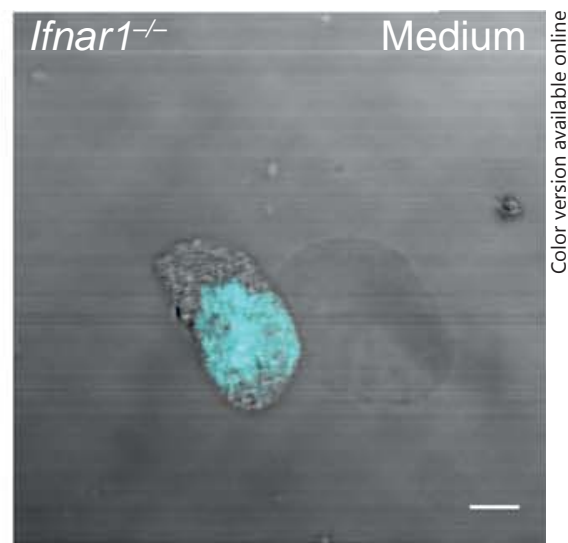

Ifnar $1^{-/-}$
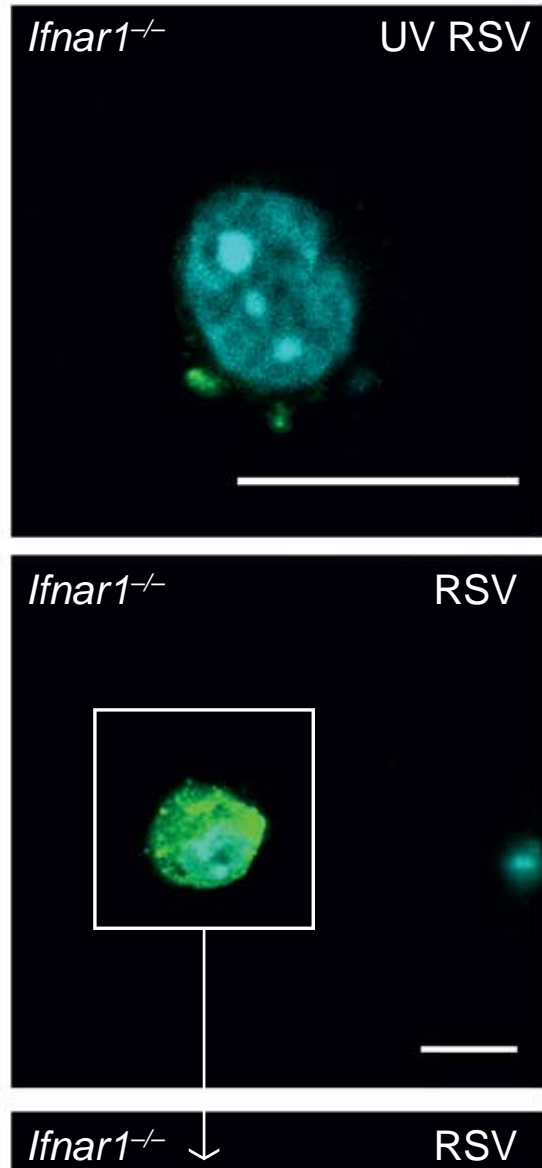


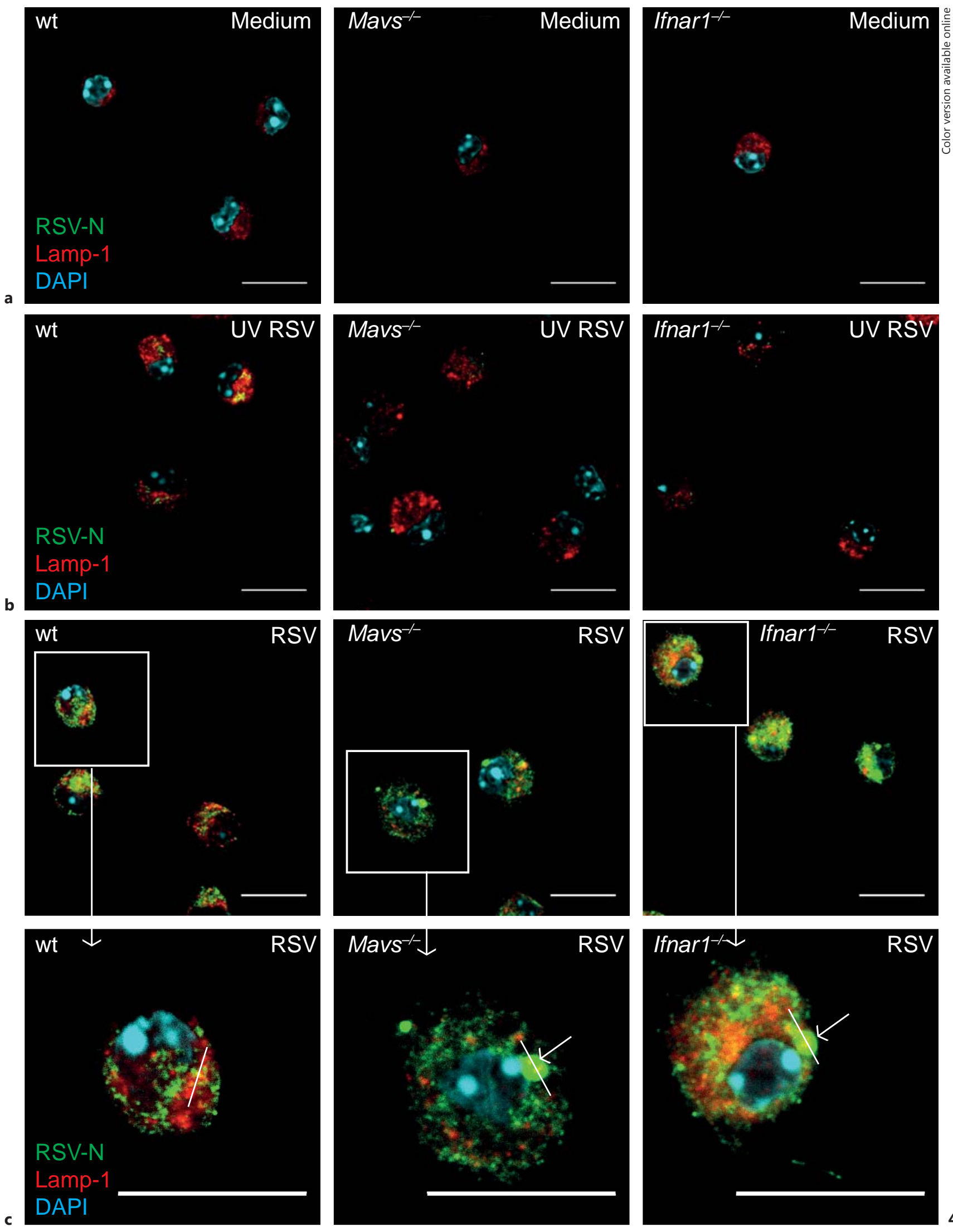

(For figure $4 d$ and legend see next page.) 
control for responsiveness, AMs were stimulated with LPS and R848, which are known to trigger TLR4 and TLR7, respectively, independently of MAVS and IFNAR. LPS and R848 stimulation resulted in the production of IL-6 and TNF- $\alpha$ from all genotypes of AMs (fig. 2c). However, Ifnar $1^{-1-}$ AMs showed decreased production of TNF- $\alpha$ after LPS stimulation and Ifnarl ${ }^{-/-}$and $\mathrm{Mavs}^{-/-}$AMs of IL-6 after LPS and R848 production compared to wt AMs (fig. 2c). This is possibly due to the importance of type I IFNs in potentiating proinflammatory responses [14].

Type I IFNs are known to induce several IFN-stimulated genes (ISGs) that interfere with viral replication. Since AMs are one of the first cells to encounter virus particles reaching the lower airways, we investigated the induction of some ISGs, i.e. viperin, OAS-1, and PKR in AMs after ex vivo exposure to RSV (MOI of 2; fig. 2d). These ISGs have all been implicated in restricting RSV replication [18-21] and were upregulated in wt AMs but not in IFNAR1- or MAVS-deficient AMs, in line with their lack of response to or induction of type I IFNs (fig. 2a, d).

\section{IFNAR and MAVS Deficiency Leads to Increased Intracellular Viral Protein Accumulation and to the Presence of Inclusion Bodies}

In order to assess if the lack of a type I IFN response and ISG induction would alter the presence and localisation of RSV proteins within AMs, fluorescence microscopy was performed. Staining for RSV proteins showed that wt AMs displayed similar punctate staining whether the cells were exposed to RSV or UV-RSV (fig. 3). In contrast, IFNAR1- and MAVS-deficient AMs showed more of a cytoplasmic staining with larger aggregated staining of viral proteins that resembled inclusion bodies (IBs;

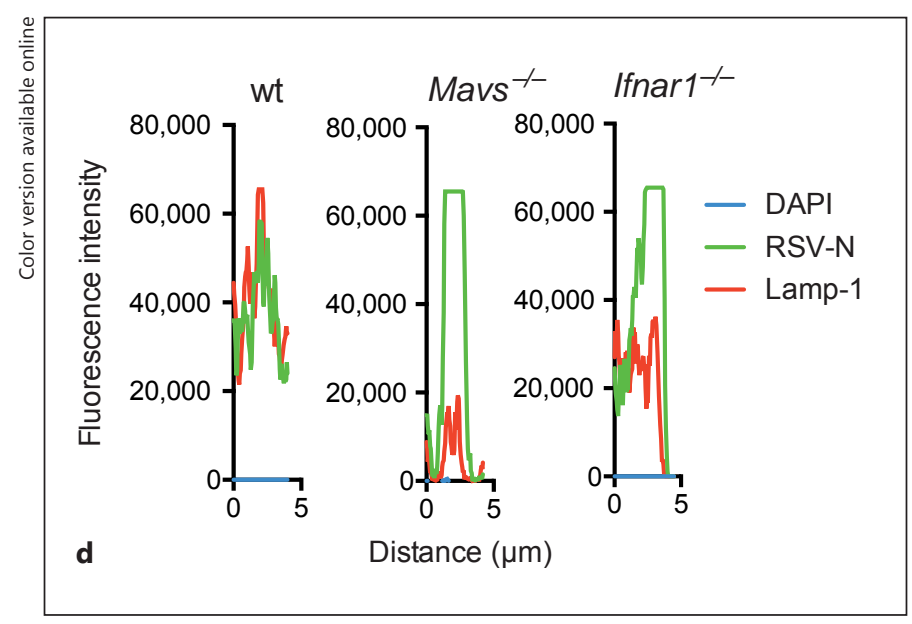

Type I IFN-Deficient AMs Can Control RSV Replication fig. 3b-c). Consistent with that notion, these large cytoplasmic bodies could be detected with an antibody against the RSV N protein, which is abundant in IBs [22], in IFNAR1- and MAVS-deficient AMs, but not in wt AMs (fig. 4). To distinguish phagolysosomes from IBs, staining for LAMP-1 was performed and demonstrated that the larger aggregates of viral proteins found in IFNAR1- and MAVS-deficient AMs did not co-stain with LAMP-1, suggesting that they were indeed IBs (fig. 4c). Furthermore, intensity analysis of the IB areas (fig. $4 \mathrm{~d}$ ) and preliminary z-stack analysis (data not shown) confirmed that IBs detected in IFNAR1- and MAVS-deficient AMs are not within phagolysosomes.

To further evaluate the presence of viral proteins in AMs, Western blot analysis using anti-RSV antibodies on lysate from UV-RSV - or RSV-exposed (MOI of 2) AMs from wt, $\mathrm{Mavs}^{-1-}$, and Ifnarl ${ }^{-1-}$ mice was performed (fig. 5). This analysis revealed proteins suggestive of RSV G, N, P, and M2-1 [23-25] in all samples (UV-RSV and RSV). However, in RSV-exposed $\mathrm{Mavs}^{-/-}$and Ifnar1 ${ }^{-/-}$ AMs, more $\mathrm{N}$ was detected (fig. 5), suggesting RSV protein production. Thus, when compared to wt AMs, lack of signalling through IFNAR1 or MAVS in AMs allows viral replication to advance further and enables the virus to form IBs.

\section{RSV Replication in AMs Is Restricted Even in the} Absence of MAVS and IFNAR Signalling

To assess the viral load in AMs exposed to RSV ex vivo, RSV L and N genes were quantified. RSV L and N genes were detected in AMs from all genotypes from 1 to $48 \mathrm{~h}$ after exposure. In the absence of type I IFNs, IFNAR1and MAVS-deficient AMs had marginally more L gene copies but significantly more $\mathrm{N}$ gene copies compared to

Fig. 4. IBs are only present in AMs deficient in MAVS and IFNAR1 signalling. Primary AMs from wt, $\mathrm{Mavs}^{-/-}$, and Ifnar1 ${ }^{-/-}$mice were exposed to MOI of 2 of RSV for $21 \mathrm{~h}$. Cells were fixed and stained using anti-RSV-N (green), anti-LAMP-1 (red), and DAPI (blue), and examined by fluorescence microscopy. Representative image of medium-exposed AMs (a) and UV-RSV-exposed AMs (b). c RSV-exposed AMs where the location of IBs is presented by white arrows. Images represent the appearance of the majority of AMs from 3 independent experiments. The scale bars represent 10 $\mu \mathrm{m}$. Monochrome images were converted to composite images using the Fiji software program (ImageJ). d Graphs indicate the pixel intensity of RSV-N, Lamp-1, and DAPI along the white line indicated on the micrographs. 
Fig. 5. The presence of RSV nucleocapsid protein is increased in MAVS- and IFNAR1-deficient AMs. Primary wt, $\mathrm{Mavs}^{-/-}$, and Ifnar1 ${ }^{-1-}$ AMs were exposed to UVRSV or RSV (MOI of 2) for $21 \mathrm{~h}$. Cell lysate was generated using RIPA buffer and then subjected to Western blot analysis. The blots were exposed to a polyclonal antiRSV antibody or to an anti- $\beta$-actin antibody. The protein standard is indicated to the right of the blot and the detected RSV proteins ( $G, N, L$, and $M$ proteins) are indicated at the right of the blots. The blots are representative of at least 3 experiments.

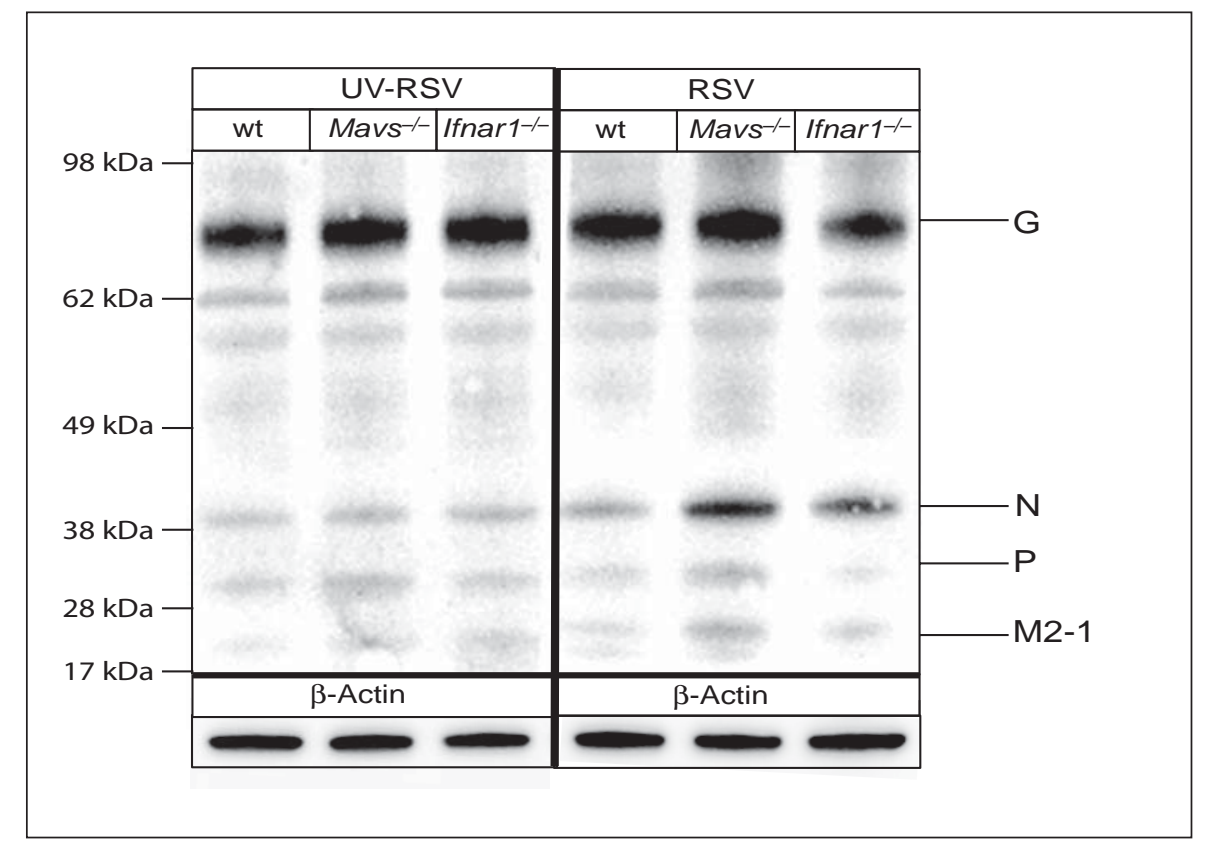

wt AMs after RSV exposure (fig. 6a; online suppl. fig. S2). We compared this to the mouse lung epithelial cell line LA4, which showed a 100- to 1,000-fold increase in L and $\mathrm{N}$ gene transcripts between 1 and $48 \mathrm{~h}$ after infection (fig. 6a). In addition, AMs or LA4 cells exposed to UVinactivated RSV (AM UV and LA4 UV) showed no increase in $\mathrm{L}$ and $\mathrm{N}$ gene copies (fig. 6a; online suppl. fig. S2). Viral load was also assessed by immunoplaque assay to quantify infectious particles produced by AMs, but no plaques could be detected (data not shown). Furthermore, using a RSV strand-specific qPCR assay [17], AMs showed similar levels of both RSV positive- and negativesense strands with a slightly higher level of positive-sense strand RSV in Ifnar ${ }^{-l-}$ AMs at $24 \mathrm{~h}$ after RSV exposure. However, LA4 cells showed a significant increase in both RSV strands (fig. 6b). Altogether, these data indicate that viral replication is slightly elevated in MAVS- and IFNAR-deficient AMs compared to wt AMs, but that RSV replicates more efficiently in the lung epithelial cells (LA4) compared to AMs.

\section{Discussion}

AMs are key cells of the lower airways in both health and disease. They have an important role in surfactant catabolism and clearing up debris as well as in sensing invading pathogens and initiating immune responses [1].
Here, we show that AMs rely on MAVS and IFNAR1 signalling to produce cytokines and chemokines, including type I IFNs, in response to RSV exposure. However, even in the absence of type I IFN production or signalling, AMs can control viral replication, indicating that they possess mechanisms independent of the canonical IFN pathway to restrict viral infection.

AMs constitute a unique subset of macrophages that develops from fetal liver monocytes, is exposed to air, and is phenotypically and functionally different from other tissue macrophages [2]. Therefore, in order to study these cells, primary AMs must be obtained from the lung, most commonly via bronchoalveolar lavage. AMs are the main producers of type I IFNs in vivo during RSV and Newcastle disease virus infection $[4,6]$. Human, ovine, and murine AMs have also been suggested to produce several cytokines and chemokines after ex vivo exposure to RSV $[4,15,23,26-31]$. We confirmed the ability of AMs to produce IFN- $\alpha$, IFN- $\beta$, TNF- $\alpha$, IL-6, CCL3, and CXCL10 after ex vivo exposure to RSV. Interestingly, several cytokines and chemokines such as CXCL1, CCL2, and IL-1 $\beta$ are known to be present in the lungs early after RSV infection [14], yet were not produced by AMs. This highlights the delicate interplay between cells within the lung in that multiple cell types are needed in order to induce a proper inflammatory response to viral infections.

Type I IFNs are important mediators to induce an antiviral state in infected and neighbouring cells, and for 


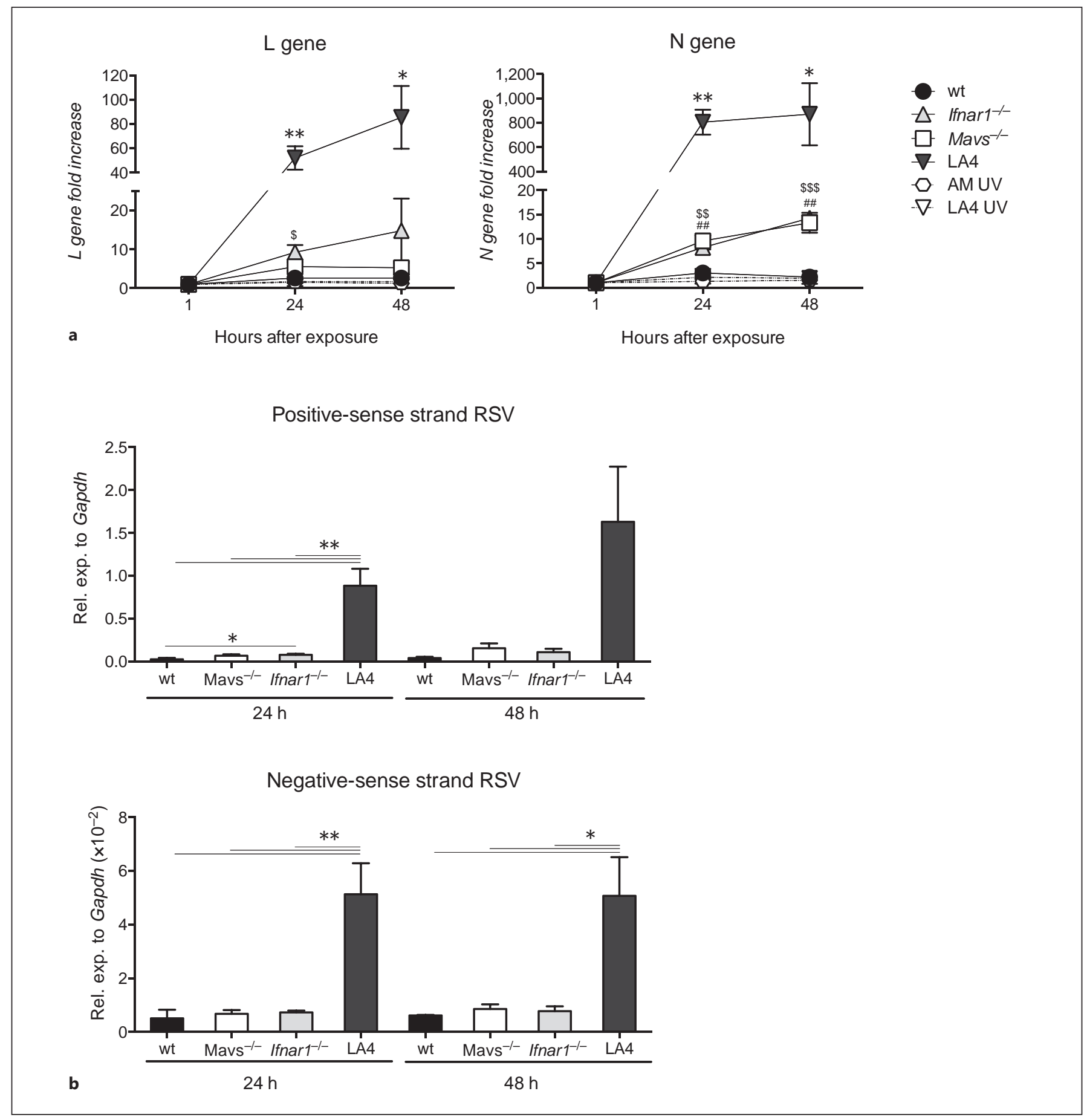

Fig. 6. Control of replication of RSV in AMs is independent of MAVS and IFNAR signalling. a AMs from wt, $\mathrm{Mavs}^{-/-}$, and Ifnar $1^{-1-}$ mice and LA4 cells were exposed to medium or MOI of 2 of UV-RSV (AM UV or LA4 UV) or RSV for 1, 24, and $48 \mathrm{~h}$. RSV $\mathrm{L}$ and $\mathrm{N}$ gene copies were determined by RT-PCR and the data are shown as fold increase over the copies present at $1 \mathrm{~h}$ after inoculation. The UV AM show pooled measurements of $\mathrm{L}$ and $\mathrm{N}$ genes from all genotypes of AMs. b Positive- and negative-sense strands of RSV were detected using RT-PCR. The data are shown as means \pm SEM of 3-4 individual RNA samples pooled from 3 independent experiments (a) and 4-6 individual RNA samples pooled from 4 independent experiments (b). Each RNA sample was extracted from 2-3 pooled individual cultures of AMs or LA4 cells. Statistical significance of differences between indicated groups was determined by unpaired Student's t test. In a '*' represents differences between LA4 and wt AM, '\$' between wt and Ifnar $1^{-l-} \mathrm{AMs}$ and '\#' between wt and $\mathrm{Mavs}^{-/-}$AMs. ${ }^{*} \mathrm{p}<0.05$; $^{* *} \mathrm{p}<0.01$; $^{* * *} \mathrm{p}<0.001$. 
the recruitment and activation of immune cells. Human AMs have been shown to be less susceptible to RSV infection than nasal and bronchial epithelial cells [32]. We therefore hypothesised that the lack of production of $\left(\mathrm{Mavs}^{-/}\right)$or signalling by (Ifnarl $\left.{ }^{-/-}\right)$type I IFNs in AMs would render these cells more susceptible to RSV replication. As expected, the $\mathrm{Mavs}^{-/-}$and Ifnarl ${ }^{-/-}$AMs were unable to induce the ISGs viperin, OAS-1, and PKR, the viral cycle was less restricted, and more viral genes and proteins were detected within the cytosol compared to wt AMs. Yet, despite the increased accumulation of viral proteins in the type I IFN-deficient AMs, RSV only underwent abortive replication, which resulted in detection of IBs. This is in contrast to cells susceptible to RSV infection, such as in the human epithelial cell line HEp2, where the virus forms filaments on the plasma membrane and IBs are less obvious $[33,34]$. How type I IFN-deficient AMs still managed to control the virus and how far into the viral life cycle is control achieved was not elucidated in this study. Nevertheless, increased presence of RSV N was detected in $\mathrm{Mavs}^{-1-}$ and Ifnarl ${ }^{-1-}$ AMs, which would suggest that initiation of viral replication occurs in these cells. It could therefore be speculated that there is an arrest either in assembly or budding. As we have not systematically compared the ability of RSV to infect multiple cell types, it remains possible that the restriction in viral replication observed in AMs is not unique. A possible reason for their ability to control viral replication might be due to particular characteristics of AMs, such as their high degree of phagocytosis. Furthermore, a growing number of cellular factors have been reported to be required for RSV replication, such as Rab11 pathway proteins, RhoA, cofilin 1, caveolin proteins, HSP90, and mitochondrial proteins. It is thus possible that lack of certain host factors [35-38] do not allow productive viral replication in AMs. Alternatively, AMs might have an altered lipid content of their cell membrane or lipid rafts compared to susceptible cells such as epithelial cells, which could prevent release of infectious virions $[39,40]$. The immunofluorescence analysis of the RSV proteins in the wt AMs showed a very similar punctate staining of RSVor UV-RSV-exposed AMs. We were not able to confirm if the RSV proteins were inside or bound to the surface of the cell. However, in the type I IFN-deficient AMs there were more RSV proteins detected within the cytosol, and the Western blot analysis and RT-PCR also showed an increase in RSV N proteins and genes. Altogether, this raises the possibility that AMs could contribute to viral clearance by mopping up virus from the airways, preventing productive infection of epithelial cells.
The ability of AMs to control RSV infection would seem to render them as ideal sensors of infection. Human epithelial cells have been suggested to be the ideal host for RSV [17]. Although RSV also replicates in the mouse lung [17, 41] and in mouse epithelial cells (LA4), AMs did not appear to support viral replication to the same degree as LA4 cells. Thus, AMs are important for initiation of the immune response in the lower airways after RSV infection and they are able to restrict viral replication even in the absence of type I IFNs. This positions them as excellent sensors of infection on the mucosal barrier.

\section{Acknowledgements}

C.J. was supported by a Career Development Award from the Medical Research Council (grant G0800311). F.J.C. was supported by an MRC grant (G1001763). M.B. was supported by Marie Curie Career Integration Grant (No. 321931) from the European Commission. S.M. and M.G. were supported by PhD Fellowships from the National Heart and Lung Institute Foundation (registered charity No. 1048073). This work was also supported by a grant from the Rosetrees Trust (M370). We thank S. Akira and Y. Kumagai (World Premier International Immunology Frontier Research Center, Osaka University, Osaka, Japan) for providing $\mathrm{Mavs}^{-/-}$mice. We thank Anabel Guedán Paredes for help and advice with the Western blot analysis. We also thank the other members of the Respiratory Infections section, especially Peter Openshaw and Chris Chiu for suggestions and critique, and the staff of the St Mary's flow cytometry facility and the animal facility. We thank Caetano Reis e Sousa for critically reading the manuscript. The Facility for Imaging by Light Microscopy (FILM) at Imperial College London is supported by funding from the Wellcome Trust (grant 104931/Z/14/Z) and BBSRC (grant BB/L015129/1).

\section{Disclosure Statement}

The authors declare no competing financial interests.

References

1 Hussell T, Bell TJ: Alveolar macrophages: plasticity in a tissue-specific context. Nat Rev Immunol 2014;14:81-93.

2 Scott CL, Henri S, Guilliams M: Mononuclear phagocytes of the intestine, the skin, and the lung. Immunol Rev 2014;262:9-24.

-3 Guilliams M, De Kleer I, Henri S, Post S, Vanhoutte L, De Prijck S, et al: Alveolar macrophages develop from fetal monocytes that differentiate into long-lived cells in the first week of life via GM-CSF. J Exp Med 2013;10:1538.

4 Goritzka M, Makris S, Kausar F, Durant LR, Pereira C, Kumagai Y, et al: Alveolar macrophage-derived type I interferons orchestrate innate immunity to RSV through recruitment of antiviral monocytes. J Exp Med 2015;212: 699-714. 
5 Tate MD, Pickett DL, van Rooijen N, Brooks AG, Reading PC: Critical role of airway macrophages in modulating disease severity during influenza virus infection of mice. J Virol 2010;84:7569-7580.

6 Kumagai Y, Takeuchi O, Kato H, Kumar H, Matsui K, Morii E, et al: Alveolar macrophages are the primary interferon-alpha producer in pulmonary infection with RNA viruses. Immunity 2007;27:240-252.

-7 Schneider C, Nobs SP, Heer AK, Kurrer M, Klinke G, van Rooijen N, et al: Alveolar macrophages are essential for protection from respiratory failure and associated morbidity following influenza virus infection. PLoS Pathog 2014;10:e1004053.

8 Pribul PK, Harker J, Wang B, Wang H, Tregoning JS, Schwarze J, et al: Alveolar macrophages are a major determinant of early responses to viral lung infection but do not influence subsequent disease development. J Virol 2008;82:4441-4448.

-9 Kopf M, Schneider C, Nobs SP: The development and function of lung-resident macrophages and dendritic cells. Nat Immunol 2014;16:36-44.

10 Borchers AT, Chang C, Gershwin ME, Gershwin LJ: Respiratory syncytial virus - a comprehensive review. Clin Rev Allergy Immunol 2013;45:331-379.

-11 Blanken MO, Rovers MM, Molenaar JM, Winkler-Seinstra PL, Meijer A, Kimpen JLL, et al: Respiratory syncytial virus and recurrent wheeze in healthy preterm infants. N Engl J Med 2013;368:1791-1799.

12 Durbin RK, Kotenko SV, Durbin JE: Interferon induction and function at the mucosal surface. Immunol Rev 2013;255:25-39.

13 McNab F, Mayer-Barber K, Sher A, Wack A, O'Garra A: Type I interferons in infectious disease. Nat Rev Immunol 2015;15:87-103.

- 14 Goritzka M, Durant LR, Pereira C, SalekArdakani S, Openshaw PJM, Johansson C: Interferon- $\alpha / \beta$ receptor signaling amplifies early pro-inflammatory cytokine production in the lung during respiratory syncytial virus infection. J Virol 2014;88:6128-6136.

15 Goritzka M, Pereira C, Makris S, Durant LR, Johansson C: $\mathrm{T}$ cell responses are elicited against respiratory syncytial virus in the absence of signalling through TLRs, RLRs and IL-1R/IL-18R. Sci Rep 2015;5:18533.

16 Lee DCP, Harker JAE, Tregoning JS, Atabani SF, Johansson C, Schwarze J, et al: CD25+ natural regulatory $\mathrm{T}$ cells are critical in limiting innate and adaptive immunity and resolving disease following respiratory syncytial virus infection. J Virol 2010;84:8790-8798.

17 Bannister R, Rodrigues D, Murray EJ, Laxton C, Westby M, Bright H: Use of a highly sensitive strand-specific quantitative PCR to identify abortive replication in the mouse model of respiratory syncytial virus disease. Virol J 2010;7:250.
18 McGillivary G, Jordan ZB, Peeples ME, Bakaletz LO: Replication of respiratory syncytial virus is inhibited by the host defense molecule viperin. J Innate Immun 2013;5:60-71.

19 Jumat MR, Huong TN, Ravi LI, Stanford R, Tan BH, Sugrue RJ: Viperin protein expression inhibits the late stage of respiratory syncytial virus morphogenesis. Antiviral Res 2015;114:11-20.

20 Behera AK, Kumar M, Lockey RF, Mohapatra SS: 2'-5' Oligoadenylate synthetase plays a critical role in interferon-gamma inhibition of respiratory syncytial virus infection of human epithelial cells. J Biol Chem 2002;277: 25601-25608.

-21 Minor RA, Limmon GV, Miller-DeGraff L, Dixon D, Andrews DMK, Kaufman RJ, et al: Double-stranded RNA-activated protein kinase regulates early innate immune responses during respiratory syncytial virus infection. J Interferon Cytokine Res 2010;30:263-272.

-22 Lifland AW, Jung J, Alonas E, Zurla C, Crowe JE, Santangelo PJ: Human respiratory syncytial virus nucleoprotein and inclusion bodies antagonize the innate immune response mediated by MDA5 and MAVS. J Virol 2012;86: 8245-8258.

23 Ravi LI, Li L, Sutejo R, Chen H, Wong PS, Tan $\mathrm{BH}$, et al: A systems-based approach to analyse the host response in murine lung macrophages challenged with respiratory syncytial virus. BMC Genomics 2013;14:190.

24 Förster A, Maertens GN, Farrell PJ, Bajorek M: Dimerization of matrix protein is required for budding of respiratory syncytial virus. J Virol 2015;89:4624-4635.

25 Munday DC, Wu W, Smith N, Fix J, Noton SL, Galloux M, et al: Interactome analysis of the human respiratory syncytial virus RNA polymerase complex identifies protein chaperones as important cofactors that promote L-protein stability and RNA synthesis. J Virol 2015;89:917-930.

26 Fach SJ, Olivier A, Gallup JM, Waters TE, Ackermann MR, Lehmkuhl HD, et al: Differential expression of cytokine transcripts in neonatal and adult ovine alveolar macrophages in response to respiratory syncytial virus or toll-like receptor ligation. Vet Immunol Immunopathol 2010;136:55-64.

27 Kolli D, Gupta MR, Sbrana E, Velayutham TS, Hong C, Casola A, et al: alveolar macrophages contribute to the pathogenesis of hMPV infection while protecting against RSV infection. Am J Respir Cell Mol Biol 2014;51:502515.

28 Demoor T, Petersen BC, Morris S, Mukherjee S, Ptaschinski C, De Almeida Nagata DE, et al: IPS-1 signaling has a nonredundant role in mediating antiviral responses and the clearance of respiratory syncytial virus. J Immunol 2012;189:5942-5953.

29 Becker S, Quay J, Soukup J: Cytokine (tumor necrosis factor, IL-6, and IL-8) production by respiratory syncytial virus-infected human alveolar macrophages. J Immunol 1991;147: 4307-4312.
0 Panuska JR, Merolla R, Rebert NA, Hoffmann SP, Tsivitse P, Cirino NM, et al: Respiratory syncytial virus induces interleukin-10 by human alveolar macrophages. Suppression of early cytokine production and implications for incomplete immunity. J Clin Invest 1995; 96:2445-2453.

-31 Franke-Ullmann G, Pförtner C, Walter P, Steinmüller C, Lohmann-Matthes ML, Kobzik L, et al: Alteration of pulmonary macrophage function by respiratory syncytial virus infection in vitro. J Immunol 1995; 154:268280.

32 Becker S, Soukup J, Yankaskas JR: Respiratory syncytial virus infection of human primary nasal and bronchial epithelial cell cultures and bronchoalveolar macrophages. Am J Respir Cell Mol Biol 1992;6:369-374.

33 Roberts SR, Compans RW, Wertz GW: Respiratory syncytial virus matures at the apical surfaces of polarized epithelial cells. J Virol 1995;69:2667-2673.

- 34 Bajorek M, Caly L, Tran KC, Maertens GN, Tripp RA, Bacharach E, et al: The Thr205 phosphorylation site within respiratory syncytial virus matrix $(\mathrm{M})$ protein modulates $\mathrm{M}$ oligomerization and virus production. J Virol 2014;88:6380-6393.

35 Utley TJ, Ducharme NA, Varthakavi V, Shepherd BE, Santangelo PJ, Lindquist ME, et al: Respiratory syncytial virus uses a Vps4-independent budding mechanism controlled by Rab11-FIP2. Proc Natl Acad Sci USA 2008; 105:10209-10214.

36 Gower TL, Pastey MK, Peeples ME, Collins PL, McCurdy LH, Hart TK, et al: RhoA signaling is required for respiratory syncytial virusinduced syncytium formation and filamentous virion morphology. J Virol 2005;79: 5326-5336.

37 Kipper S, Hamad S, Caly L, Avrahami D, Bacharach E, Jans DA, et al: New host factors important for respiratory syncytial virus (RSV) replication revealed by a novel microfluidics screen for interactors of matrix (M) protein. Mol Cell Proteomics 2015; 14:532543.

38 Brock SC, Goldenring JR, Crowe JE: Apical recycling systems regulate directional budding of respiratory syncytial virus from polarized epithelial cells. Proc Natl Acad Sci USA 2003;100:15143-15148.

-39 Chang TH, Segovia J, Sabbah A, Mgbemena V, Bose S: Cholesterol-rich lipid rafts are required for release of infectious human respiratory syncytial virus particles. Virology 2012 422:205-213.

40 McCurdy LH, Graham BS: Role of plasma membrane lipid microdomains in respiratory syncytial virus filament formation. J Virol 2003;77:1747-1756.

41 Loebbermann J, Thornton H, Durant L, Sparwasser T, Webster KE, Sprent J, et al: Regulatory $\mathrm{T}$ cells expressing granzyme $\mathrm{B}$ play a critical role in controlling lung inflammation during acute viral infection. Mucosal Immunol 2012;5:161-172. 